\title{
Repetition rate multipliers for efficient implementation of multiphoton and pump-probe fluorescence microscopy
}

\author{
Jie Chen, Cheng Hao Chien, Hsuan Shao Kuo, Chen-Yuan Dong
}

Department of Physics, National Taiwan University, Taipei 106, Taiwan

\begin{abstract}
With the advances in pulsed laser systems, microscopic imaging techniques such as multiphoton and pumpprobe fluorescence microscopy have developed into effective tools for investigating intensity and time-resolved phenomena inside biological systems. However, pulsed lasers used in these techniques usually are commercial systems with repetition frequencies of around $80 \mathrm{MHz}$. While these systems have proven to be adequate for multiphoton and pump-probe microscopic imaging applications, the temporal separation of the laser pulse train (around $12.5 \mathrm{~ns}$ ) is long compared to the fluorescence lifetimes of many common fluorescence species. In this work, we present the designs of repetition rate multipliers based on passive optical components that can be used to increase the efficiency in multiphoton and pump-probe fluorescence microscopy. Depending on the lifetime of fluorescence molecules under investigation, the passive repetition rate multiplier can increase the duty cycle of multiphoton or pump-probe microscopy up to fourfold.
\end{abstract}

Key words: Multiphoton - pumb-probe - fluorescence-microscopy

\section{Introduction}

In recent years, novel microscopic imaging techniques based on multiphoton excitation and pump-probe methodology added to the repertoire of techniques available for biological fluorescence imaging applications. In multiphoton fluorescence microscopy, the fluorescence species of interest is excited to the excited state by the simultaneous absorption of two or more photons. Unlike conventional techniques based on single-photon excitation, the non-linear excitation confines fluorescence excitation to near the focal volume. As a result, confocal-like imaging quality is achieved without the use of confocal pinholes. Furthermore, since sample excitation occurs only at the focal volume, specimen photodamage is also limited to the focus. Finally, since the near-infrared photons used for sample excitation are absorbed and

Received 8 April 2003; accepted 10 August 2003.

Correspondence to: C.-Y. Dong

Fax: ++886-2-2363-9984

E-mail: cydong@phys.ntu.edu.tw scattered less by biological specimens, multiphoton excitation microscopy can penetrate deeper into biological structures without the use of disruptive histological procedures [1-3]. In the life sciences, multiphoton fluorescence microscopy has found wide applications in diverse disciplines such as deep-tissue imaging, neurobiology, and developmental biology [4-9]. Indeed, multiphoton microscopy has become one of the most important fluorescence imaging techniques in modern optical microscopy. On the other hand, pump-probe fluorescence microscopy represents a more recent attempt to obtain time-resolved biological images with similar depth discrimination capability. In this novel technique, two pulsed laser systems with different repetition frequencies are focused and spatially overlapped at the fluorescent sample. The wavelength of one of the lasers (the pump) is chosen to excite the sample fluorescence while the other laser's wavelength (the probe's) is chosen to induce stimulated emission from the excited state fluorophores. Due to the difference in repetition frequencies of the two lasers, the fluorophore excited state lifetime is continuously sampled at different times following the excitation pulse. In the frequency-domain, the difference in the pump and probe lasers' repetition frequencies results in a sinusoidal fluorescence signal at the difference frequency and its harmonics. As in frequency-domain fluorescence microscopy, the amplitude and phase of the cross-correlation signal can be used to achieve time-resolved microscopic imaging. A major advantage of pump-probe microscopy is that the high-frequency, lifetime information of the fluorescence species can be determined from the low-frequency, cross-correlation signals without the use of high speed photodetectors. Furthermore, since the pump-probe signal depends on the spatial overlap of the two lasers, the cross-correlation signal originates primarily from the focal volume where the pump and probe lasers both have high intensities. As a result, as in multiphoton microscopy, pump-probe microscopy can achieve confocal-like imaging image quality without using apertures [10-13]. The basic principles of two-photon (as a representative of multiphoton processes) and pump-probe fluorescence excitation are illustrated in fig. 1. 

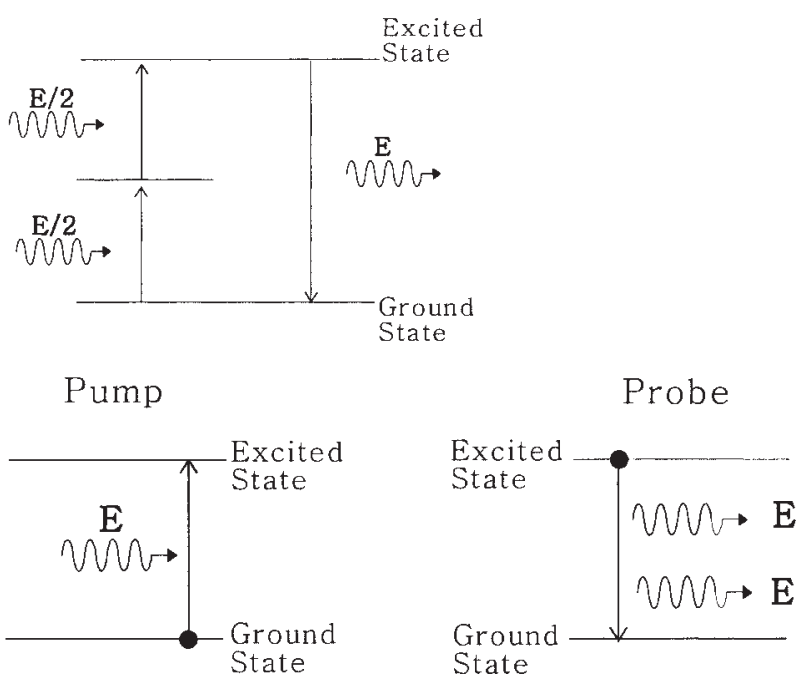

Fig. 1. Principles of two-photon (top) and pump-probe (bottom) excitation.

\section{Duty cycle in pulsed laser fluorescence microscopy}

In most laboratories conducting scanning microscopy using pulsed laser sources, the commercial laser excitation sources have repetition frequencies around $80 \mathrm{MHz}$. A repetition frequency of $80 \mathrm{MHz}$ translates to a temporal separation of the laser pulses of $12.5 \mathrm{~ns}$. Depending on the molecules under study, $12.5 \mathrm{~ns}$ can be much longer than the molecular fluorescence lifetime. For example, rhodamine and fluorescein, two of the commonly used fluorophores in fluorescence microscopy have lifetimes around 2.0 and $4.5 \mathrm{~ns}$, respectively [14]. Assuming a single exponential model of $e^{-t / \tau}$ (where $\tau$ is the fluorescence lifetime) and defining the effective detection period as the time from the initiation of the fluorescence decay to when $90 \%$ of the fluorescence has decayed, the duty cycle of fluorescence detection is $37 \%$ and $83 \%$ for rhodamine and fluorescein, respectively. For fluorophores with a lifetime of $1 \mathrm{~ns}$, the duty cycle is merely $18 \%$. As illustrated in fig. 2 , in order to maximize the detection efficiency in multiphoton or pump-probe studies, it is important to match the repetition frequency of the pulsed laser systems used to the lifetime of the fluorescent molecules.

For multiphoton or pump-probe spectroscopy, the low detection duty cycle is less problematic. The focused laser spot can park within the specimen and the signal photons can be integrated to provide information of the photophysical properties of the specimen. However, for microscopic imaging applications, the low duty cycle can lead to inadequate detection of spatially resolved structures. The reason for this difficulty is the relatively short pixel residence time of optical microscopy. In typical applications of multiphoton or pump-probe microscopy, spatially resolved information of the fluorescence specimens is acquired by scanning a focused spot of the laser beams across the specimen. The sample image is ac-
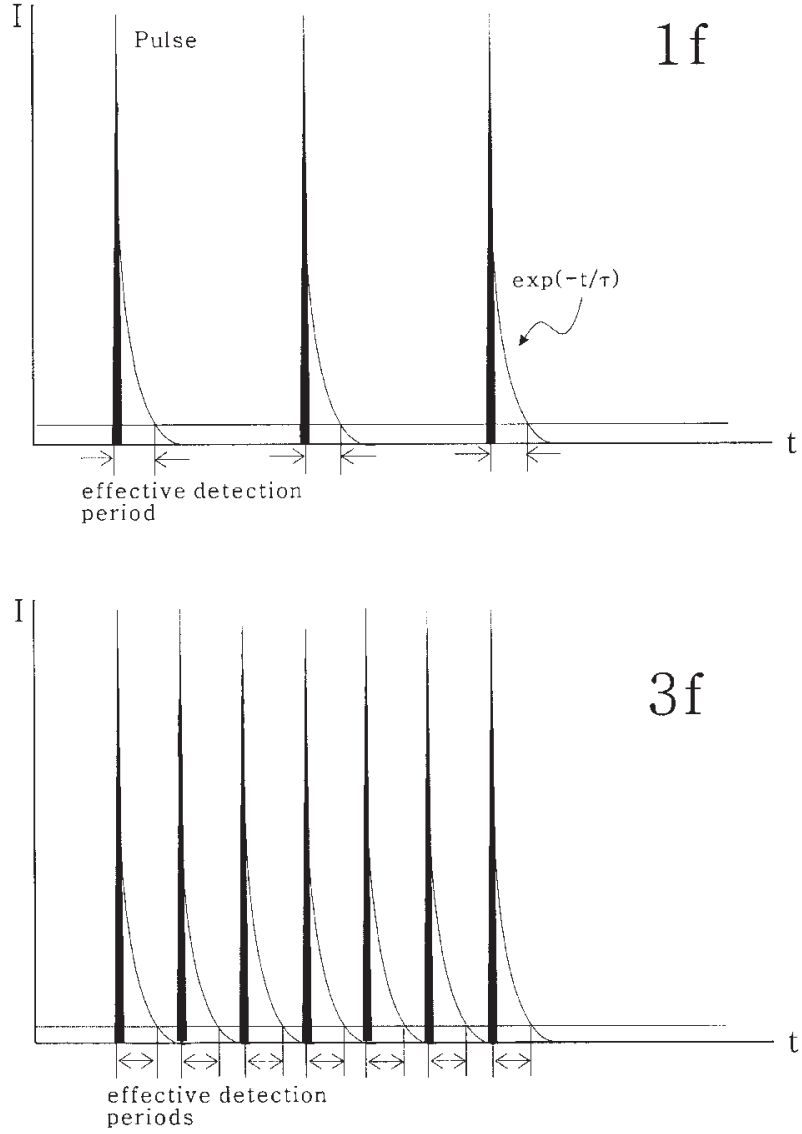

Fig. 2. Effects of laser pulse spacing on fluorescence detection efficiency.

quired by collecting sample fluorescence emission in a point-by-point fashion. To acquire an image in a timely fashion, the laser spot can only park at one point within the specimen for a short time. For example, for a 256 by 256 pixels image to be acquired within one second, the laser spot can only park at a point within the specimen for about $15 \mu \mathrm{s}$. For excitation using an $80 \mathrm{MHz}$ pulsed source, a pixel residence time only permits about 1221 laser pulses for pixel excitation. In cases where the fluorophores' lifetime is much less than $12.5 \mathrm{~ns}$, increasing the laser repetition frequency will increase the number of pulses impinging upon the pixel and will lead to an improvement in the signal to noise ratio of the fluorescence image. For example, the multiphoton excitation efficiency of fluorophores with a 1.0 ns lifetime may be enhanced when the repetition rate of the excitation source is increased from 80 to $320 \mathrm{MHz}$ (temporal pulse separation of $3.125 \mathrm{~ns}$ ). Assuming that the fluorescence emission is photon-limited, one would expect, for the same pixel residence time, the 4-fold increase in the number of pulses will lead to an increase in the $\mathrm{S} / \mathrm{N}$ ratio by a factor of two. Naturally, the choice of optimal repetition rate will depend upon the fluorophores' fluorescence lifetime. In general, it is best to avoid the temporal overlap of emission photons from different 
excitation pulses. This issue is less critical for intensity imaging. However, for time-resolved imaging, crosstalk between fluorescence photons originating from different excitation pulses will lead to inaccuracy in fluorescence lifetime determination. In the previous analysis, we arbitrarily set the $90 \%$ decay point as the point where the next excitation pulse can arrive to excite the fluorophores with minimal cross-talks. Since the exponential decay function continues to infinity, any set-point for determining the end of fluorescence emission from an excitation pulse will introduce error in fluorescence lifetime determination.

\section{Passive repetition rate multiplier designs}

An increase in excitation laser repetition rate may be achieved by using passive optical components such as beam splitters and mirrors. Considering that the fluorescence lifetimes of most fluorophores are in the ns range, increasing the repetition frequencies of commercially available pulsed laser systems with repetition frequencies from $80 \mathrm{MHz}$ to $160 \mathrm{MHz}$ (pulse separation $6.25 \mathrm{~ns}$ ), $240 \mathrm{MHz}$ (pulse separation $4.17 \mathrm{~ns}$ ), and $320 \mathrm{MHz}$ (pulse separation $3.13 \mathrm{~ns}$ ) can increase the detection efficiency for the same pixel residence time or reduce the image acquisition time in achieving the same $\mathrm{S} / \mathrm{N}$ ratio. In the designs of these passive repetition rate multipliers, it is important to design them with appropriate path length to ensure equal temporal separation between succeeding pulses.

In an arrangement shown in fig. 3, two beam splitters and two mirrors may be combined to form a repetition rate doubler. In this configuration, beam splitter 1 (BS1) splits the incoming laser pulse train into two paths. Upon reflection by the two mirrors (M1 and M2), the beam reflected by and transmitted through BS1 is combined at the second beam splitter (BS2). With proper choice of the path length difference $\left(d_{2 f}=c /(4 f), c\right.$ is the speed of light and $f$ is the input laser repetition frequency), outputs $I(2 f, 1)$ and $I(2 f, 2)$ will each contain a pulse train with a repetition frequency of $2 f$. In this arrangement,

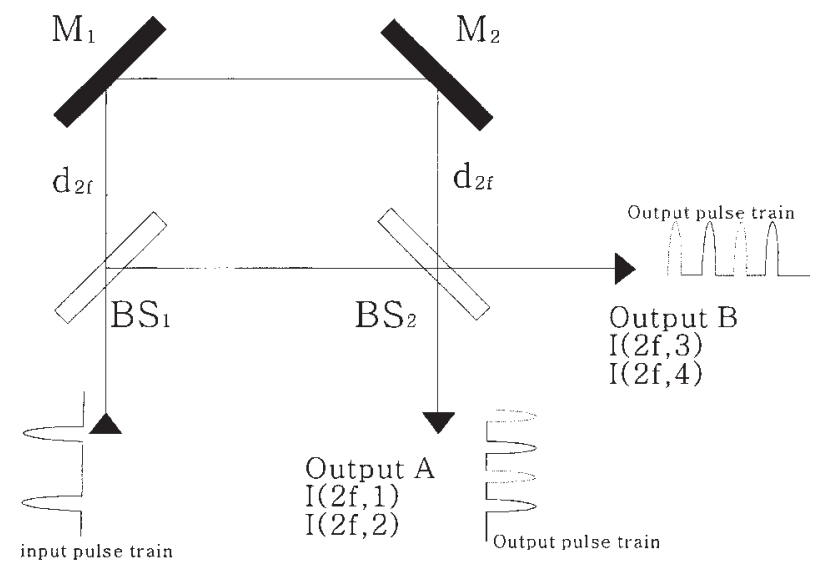

Fig. 3. A passive repetition rate doubler. we can choose the transmission ratios of BS1 $\left(T_{1}\right)$ and BS2 $\left(T_{2}\right)$ such that each pulse at the two outputs will have the same energy. To be specific, the non-delayed and delayed pulse ratio will be $\left(1-T_{1}\right)\left(1-T_{2}\right)$ and $T_{1} T_{2}$ at output A. At output B, the respective ratios are $\left(1-T_{1}\right) T_{2}$ and $T_{1}\left(1-T_{2}\right)$. In this case, a choice of using $50 / 50$ beam splitter at BS1 and BS2 $\left(T_{1}=T_{2}=0.5\right)$ will result in each of the pulses at outputs $\mathrm{A}$ and $\mathrm{B}$ having 0.25 of the incident laser pulse energy.

In the case that higher repetition frequencies from the base frequency $f$ are needed, the $2 f$ design illu-
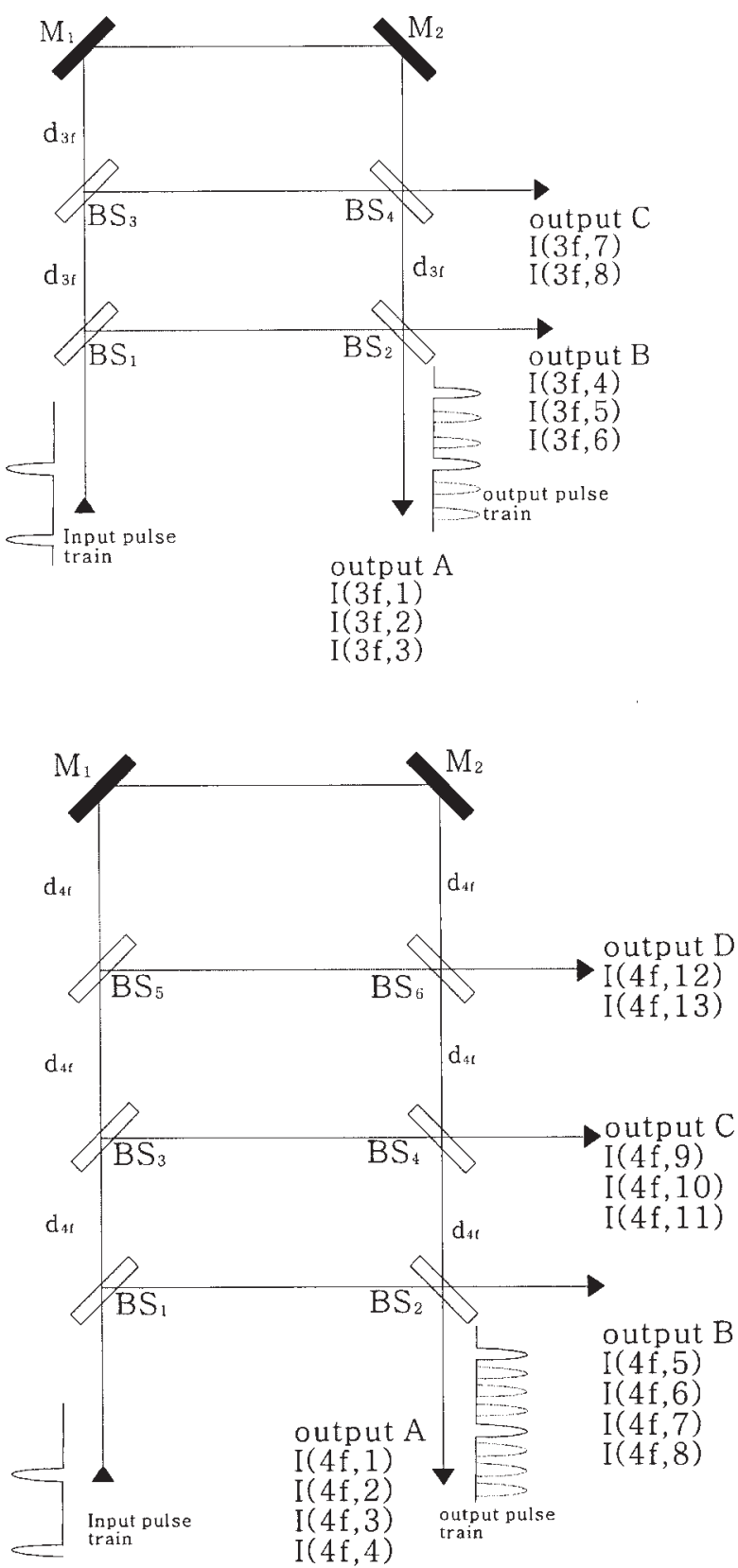

Fig. 4. Repetition rate tripler (top) and quadrupler (bottom) configurations. 
strated in fig. 3 may be expanded. For example, as shown in fig. 4 , to generate pulse trains at $3 f$ and $4 f$, two and four additional beam splitters need to be respectively added. The additional sets of beam splitters are intended to create additional reflection and transmission surfaces. In higher repetition rate systems, it becomes less obvious of the choices of the optical properties of the beam splitting elements. In the case of the $3 f$ design, multiple outputs $(\mathrm{A}, \mathrm{B}$, and $\mathrm{C})$ are possible. Since it is not possible to generate $3 f$ pulses at all outputs, we choose to generate a pulse at output A where the $3 f$ pulses are evenly spaced in time and each having the same pulse energy. In table 1 , we list the pulse outputs generated by the multiple reflection and transmission processes for the $3 f$ and $4 f$ multipliers. The problem now becomes one solving the various output terms in generating pulses with equal energy and at the desired repetition frequencies. While this may appear to be complex, there is an iterative method in which $T_{i}$, the transmission of a particular beam splitter, can be derived. For example, in the $3 f$ design, if the goal is to generate $3 f$ pulses at output A, the reflected beams from $\mathrm{BS}_{4}$ and $M_{2}$ must have equal intensity along the path between $\mathrm{BS}_{4}$ and $\mathrm{BS}_{2}$. This limits the transmission ratios of $\mathrm{BS}_{3}$ and $\mathrm{BS}_{4}$ to be 0.5 . Furthermore, if we select $T_{1}$ and $T_{2}$ to have the same value, then by requiring all reflected and transmitted pulses to have the same energy, $T_{1}$ and $T_{2}$ may be solved to be $2 / 3$. As a result, $I(3 f, 1), I(3 f, 2)$, and
$I(3 f, 3)$ will each have $1 / 9$ of the incident intensity. To ensure even temporal spacing of the three pulses, one needs to select the distance between the optical components to match the desired repetition frequencies. In the $3 f$ case, twice the time required for an $80 \mathrm{MHz}$ pulse train to traverse $d_{3 f}$ must be equal to $4.17 \mathrm{~ns}$ $\left(=2 d_{3 f} / c\right)$ such that $d_{3 f}$ is about 0.6 meter. The same reasoning we applied to the $3 f$ design can also be applied to the $4 f$ design. The choice of transmission ratios from $T_{1}$ to $T_{6}$ may be constructed by requiring pulse transmission and reflection, respectively, from $\mathrm{BS}_{5}$ and $\mathrm{BS}_{6}$ to be equal (i.e. $T_{5}=T_{6}=0.5$ ) and succeeding choices of the beam splitters' optical properties can be made by requiring the pulses at output $\mathrm{A}$ to have the same energy. However, in this case, $d_{4 f}$, the distance between optical components, is set by the requirement $2 d_{4 f} / c=3.13 \mathrm{~ns}$ (for $f=80 \mathrm{MHz}$ ). In this case, output A contains four equally spaced pulses $I(4 f, 1)$ to $I(4 f, 4)$ each with $1 / 16$ of the incident pulse energy. The results for the $3 f$ and $4 f$ repetition rate multipliers are tabulated in table 1 .

Our design can be generalized to the $n$th case where a repetition rate of $n f$ is desired. From the discussion above, it can be seen that in the generalized case, $(n-1)$ pairs of beam splitters (with the same transmission properties) and a pair of reflective mirrors are needed to generate the $n f$ pulse train. In addition, the transmission properties of the succeeding beam splitting pairs are $(1-1 / 2),(1-1 / 3),(1-1 / 4) \ldots(1-1 / n)$ and $d_{n f}$ the

Table 1. Repetition frequency multiplied outputs for $3 f$ and $4 \boldsymbol{f}$ designs.

\begin{tabular}{|c|c|c|c|c|c|}
\hline $3 f$ & Output & $\begin{array}{l}\text { Normalized } \\
\text { Intensity }\end{array}$ & $4 f$ & Output & $\begin{array}{c}\text { Normalized } \\
\text { Intensity }\end{array}$ \\
\hline$I(3 f, 1)$ & $T_{1} T_{2} T_{3} T_{4}$ & $1 / 9$ & $I(4 f, 1)$ & $\left(1-T_{1}\right)\left(1-T_{2}\right)$ & $1 / 16$ \\
\hline$I(3 f, 2)$ & $T_{1} T_{2}\left(1-T_{3}\right)\left(1-T_{4}\right)$ & $1 / 9$ & $I(4 f, 2)$ & $T_{1} T_{2}\left(1-T_{3}\right)\left(1-T_{4}\right)$ & $1 / 16$ \\
\hline$I(3 f, 3)$ & $\left(1-T_{1}\right)\left(1-T_{2}\right)$ & $1 / 9$ & $I(4 f, 3)$ & $T_{1} T_{2} T_{3} T_{4}\left(1-T_{5}\right)\left(1-T_{6}\right)$ & $1 / 16$ \\
\hline$I(3 f, 4)$ & $T_{1}\left(1-T_{2}\right) T_{3} T_{4}$ & $1 / 18$ & $I(4 f, 4)$ & $T_{1} T_{2} T_{3} T_{4} T_{5} T_{6}$ & $1 / 16$ \\
\hline$I(3 f, 5)$ & $T_{1}\left(1-T_{2}\right)\left(1-T_{3}\right)\left(1-T_{4}\right)$ & $1 / 18$ & $I(4 f, 5)$ & $\left(1-T_{1}\right) T_{2}$ & $3 / 16$ \\
\hline$I(3 f, 6)$ & $\left(1-T_{1}\right) T_{2}$ & $2 / 9$ & $I(4 f, 6)$ & $T_{1} T_{2}\left(1-T_{3}\right)\left(1-T_{4}\right)$ & $1 / 48$ \\
\hline$I(3 f, 7)$ & $T_{1} T_{3}\left(1-T_{4}\right)$ & $1 / 6$ & $I(4 f, 7)$ & $T_{1}\left(1-T_{2}\right) T_{3} T_{4}\left(1-T_{5}\right)\left(1-T_{6}\right)$ & $1 / 48$ \\
\hline \multirow[t]{7}{*}{$I(3 f, 8)$} & $T_{1}\left(1-T_{3}\right) T_{4}$ & $1 / 6$ & $I(4 f, 8)$ & $T_{1}\left(1-T_{2}\right) T_{3} T_{4} T_{5} T_{6}$ & $1 / 48$ \\
\hline & & & $I(4 f, 9)$ & $T_{1}\left(1-T_{3}\right) T_{4}$ & $1 / 6$ \\
\hline & & & $I(4 f, 10)$ & $T_{1} T_{3}\left(1-T_{4}\right)\left(1-T_{5}\right)\left(1-T_{6}\right)$ & $1 / 24$ \\
\hline & & & $I(4 f, 11)$ & $T_{1} T_{3}\left(1-T_{4}\right) T_{5} T_{6}$ & $1 / 24$ \\
\hline & & & $I(4 f, 12)$ & $T_{1} T_{3}\left(1-T_{5}\right) T_{6}$ & $1 / 8$ \\
\hline & & & $I(4 f, 13)$ & $T_{1} T_{3} T_{5}\left(1-T_{6}\right)$ & $1 / 8$ \\
\hline & $\begin{array}{l}T_{1}=T_{2}=2 / 3 \\
T_{3}=T_{4}=0.5\end{array}$ & & & $\begin{array}{l}T_{1}=T_{2}=3 / 4 ; \\
T_{3}=T_{4}=2 / 3 ; T_{5}=T_{6}=0.5\end{array}$ & \\
\hline
\end{tabular}




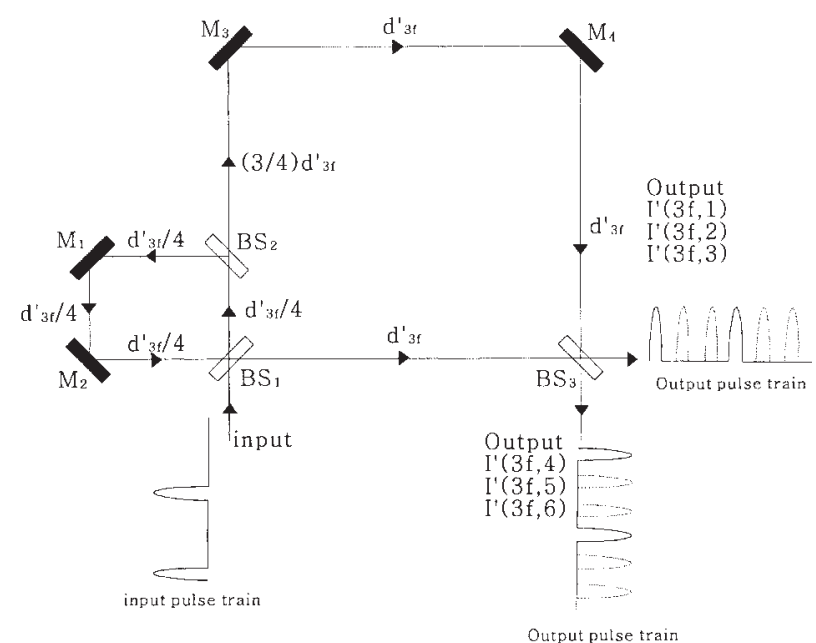

Fig. 5. Alternative configuration of a repetition frequency tripler.

distance between the pairs of optical components is determined by $2 d_{n f} / c=1 /(n f)$.

The arrangements demonstrated above are not the only possibilities. For example, one may use the configuration shown in fig. 5 to generate output with a repetition frequency three times that of the fundamental. In this configuration, three beam splitters and four mirrors are used. As in the cascade designs proposed in figs. 3 and 4, this design functions by the appropriate choices of the properties of $\mathrm{BS}_{1}-\mathrm{BS}_{3}$. However, the design illustrated in fig. 5 is more complex in analysis. Upon incident on $\mathrm{BS}_{1}$ and $\mathrm{BS}_{2}$, the laser pulse is split such that part of the energy traverses in the clock-wise loop while the remaining portion travels in the counter clock-wise path. In the counter clock-wise loop involving mirrors $\mathrm{M}_{1}$ and $\mathrm{M}_{2}$, the pulse, in theory, travels in the loop infinitely and loses part of its energy every time it is incident upon $\mathrm{BS}_{1}$ and $\mathrm{BS}_{2}$. At that point, the escaped pulse may exit the loop by further interaction with $\mathrm{BS}_{3}$. Since the counter clock-wise loop involves, in theory, infinite reflection and transmission events, the analysis for obtaining the beam splitters' transmission coefficients require numerical methods. First, we chose $T_{3}=0.5$ in order to match the intensity of outputs $I^{\prime}(3 f, 1), I^{\prime}(3 f, 2), I^{\prime}(3 f, 3)$ to that of $I^{\prime}(3 f, 4) I^{\prime}(3 f, 5)$ $I^{\prime}(3 f, 6)$. Let's focus on $I^{\prime}(3 f, 1), I^{\prime}(3 f, 2)$, and $I^{\prime}(3 f, 3)$. After summing the contributions of the multiply reflecting and transmitting beams, we arrive at the expressions for $I^{\prime}(3 f, 2)$, and $I^{\prime}(3 f, 3)$ :

$$
\begin{aligned}
I^{\prime}(3 f, 2)= & T_{1}\left[\left(T_{2}+T_{1}\left(1-T_{1}\right)\left(1-T_{2}\right)^{2}\right] /\right. \\
& {\left[2\left\{1-\left(1-T_{2}\right)^{3}\left(1-T_{1}\right)^{3}\right\}\right], } \\
I^{\prime}(3 f, 3)= & T_{1}\left(1-T_{2}\right)\left[T_{1}+T_{2}\left(1-T_{2}\right)\left(1-T_{1}\right)^{2}\right] / \\
& {\left[2\left\{1-\left(1-T_{2}\right)^{3}\left(1-T_{1}\right)^{3}\right\}\right] . }
\end{aligned}
$$

By equating (1) and (2), the set of $T_{1}$ and $T_{2}$ satisfying eqs. (1) and (2) may be found. Next, the pairs of $T_{1}$ and $T_{2}$ can be used to evaluate, for example, $I^{\prime}(3 f, 2)$. By finding those values of $T_{1}$ and $T_{2}$ that make $I^{\prime}(3 f, 2)=1 / 6$, we also constrained $I^{\prime}(3 f, 1)$ and $I^{\prime}(3 f, 3)$ to be $1 / 6$ of the incident pulse energy. The symmetry in this design then would limit $I^{\prime}(3 f, 4), I^{\prime}(3 f, 5)$, and $I^{\prime}(3 f, 6)$ to be $1 / 6$ of the incident pulse energy also. By numerical analysis, the values of $T_{1}=0.724$ and $T_{2}=0.382$ will generate $I^{\prime}(3 f, 1), I^{\prime}(3 f, 2)$, and $I^{\prime}(3 f, 3)$ to be $1 / 6$ of the incident intensity. Therefore, by choosing the distance $d_{3 f}^{\prime}=c / 3 f$, two separate pulse trains (equal pulse energy per pulse) at three times the repetition frequency can then be generated.

\section{Discussion}

By increasing the repetition frequencies using passive optical components, the detection duty cycle can be greatly increased. Since most fluorescent molecules have lifetimes in the ns range, we focused on the designs and applications of frequency doubler, tripler, and quadrupler to the commonly available $80 \mathrm{MHz}$ pulsed laser systems. For applications in multiphoton microscopy, the designs described here can increase the detection efficiency. However, for frequency-domain, pump-probe applications, two sets of independent repetition rate multipliers (one each for the pump and probe lasers) will be needed. By imaging with lasers of increased repetition frequencies, improved $\mathrm{S} / \mathrm{N}$ and/or shortened image acquisition can be achieved.

There are a number of issues to be noted with the passive repetition rate multipliers described here. First, in achieving the higher repetition frequencies, loss in the excitation power may result. For example, in the $3 f$ design (fig. 4), only 1/9 (per pulse) of the incident pulse energy is available for tripled repetition frequency excitation. In the $4 f$ design, only $1 / 16$ of the pulse energy can be used for sample excitation. Taking into account the increase in repetition frequencies, only $1 / 3$ ( $3 f$ design in fig. 4) and 1/4 (4f design in fig. 4) of the incident energy are used for sample excitation at the increased repetition frequencies. Nonetheless, with the available power in modern pulsed laser systems, the energy lost may not affect excitation efficiency. For example, a commercial titanium-sapphire laser used for multiphoton excitation can have average power near $2 \mathrm{~W}$. Used in conjunction with a $4 f$ multiplier, an average laser power of $500 \mathrm{~mW}$ is still available for fluorophore excitation. Furthermore, it should be noticed that repetition frequencies other than the intended repetition frequency may be generated. For example, in both the $3 f$ and $4 f$ designs (fig. 4 ), a $2 f$ source is generated at outputs $\mathrm{C}(3 f)$ and $\mathrm{D}(4 f)$, each pulse having respective energy $1 / 6$ and $1 / 8$ that of the incident pulse. In these designs, other pulse trains are generated (output B for $3 f$ and outputs B, C for $4 f$ ). However, since the pulse energy in these pulse trains is not uniform, we will not consider using them for microscopic imaging applications. Finally, one should notice that some of the transmission coefficients presented here in these designs are not typical items and may need to be specially synthe- 
sized. For example, as table 1 shows, the cascade design ( $3 f$ and $4 f$ ) requires a beam splitter with transmission coefficient of $2 / 3$. To the best of our knowledge, this item would not be of the standard type and may need to be specially synthesized.

\section{Conclusion}

By combining passive optical components of specially designed beam splitting elements and highly reflecting mirrors, typical pulsed laser systems operating at around $80 \mathrm{MHz}$ may be repetition frequency multiplied to generate uniform pulse trains at higher repetition rates. The generation of these higher frequencies is important for multiphoton, pump-probe and other fluorescence imaging techniques using pulsed laser systems. The temporal pulse separation of $80 \mathrm{MHz}$ lasers is long compared to the lifetimes of many fluorescent molecules. By implementing the passive repetition frequency multipliers described in this work, the detection efficiency of fluorescence microscopy based on pulsed laser systems may be increased. Shorter image acquisition time and images with improved $\mathrm{S} / \mathrm{N}$ ratio can result.

Acknowledgement. We would like to acknowledge the support of NSC 90-2112-M-002-054 and NSC 91-2112-M-002-048 from the National Science Council (Taiwan).

\section{References}

[1] Denk W, Strickler JH, Webb WW: Two-photon laser scanning fluorescence microscopy. Science 248 (1990) 73-76

[2] So PTC, Dong CY, Masters BR, Berland KM: Twophoton excitation fluorescence microscopy. Ann. Rev. Biomed. Eng. 2 (2000) 399-429
[3] Centonze VE, White JG: Multiphoton excitation provides optical sections from deeper with scattering specimens than confocal imaging. Biophys. J. 75 (1998) 20152024

[4] Denk W, Delaney KR, Gelperin A, Kleinfeld D, Strobridge BW, Tank DW, Yuste R: Anatomical and functional imaging of neurons using 2-photon laser scanning microscopy: J. Neurosci. Medthods 54 (1994) $151-62$

[5] Svoboda K, Denk W, Kleinfeld D, Tank DW: In vivo dendritic calcium dnamics in neocortical pyramidal neurons. Nature 385 (1997) 161-165

[6] Yuste R, Denk W: Dendritic spines as basic functional units of neuronal integration. Nature 375 (1995) 682684

[7] Squirrell JM, Wokosin DL, White JG, Bavister BD: Long-term two-photon fluorescence imaging of mammalian embryos without compromising viability. Nat. Biotechnol. 17 (1999) 763-767

[8] Masters BR, So PTC, Gratton E: Multiphoton excitation fluorescence microscopy and spectroscopy of in vivo human skin. Biophys. J. 72 (1997) 2405-2412

[9] Piston DW, Masters BR, Webb WW: Three-dimensionally resolved $\mathrm{NAD}(\mathrm{P}) \mathrm{H}$ cellular metabolic redox imaging of the in situ cornea with two-photon excitation laser scanning microscopy. J. Microsc. 178 (1995) 20-27

[10] Dong CY, So PTC, French T, Gratton E: Fluorescence lifetime imaging by asynchronous pump-probe microscopy. Biophys. J. 69 (1995) 2234-2242

[11] Dong CY, So PTC, Buehler C, Gratton E: Spatial resolution in pump-probe microscopy. Optik 106 (1997) $7-14$

[12] Dong CY, Buehler C, So PTC, French T, Gratton E: Implementation of intensity modulated laser diodes in timeresolved, pump-probe fluorescence microscopy. Appl. Opt. 40 (2001) 1109-1115

[13] Buehler C, Dong CY, So PTC, French T, Gratton E: Time-resolved polarization imaging by pump-probe (stimulated emission) fluorescence microscopy. Biophys. J. 79 (2000) 536-549

[14] Lakowicz, JR: Principles of Fluorescence Spectroscopy $2^{\text {nd }}$ Ed. Kluwer Academic/Plenum Publishers, New York 1999 